\title{
Lens-free and portable quantitative phase microscope using a dual-pinhole aperture
}

\author{
Yujie LU1 ${ }^{1}$, Yunhui Liu ${ }^{1}$,, Zerui Wang ${ }^{1}$, and Fan Zheng ${ }^{1}$ \\ ${ }^{1}$ Department of Automation and Mechanical Engineering, \\ The Chinese University of Hong Kong, Shatin, N.T. 999077, Hong Kong, China
}

\begin{abstract}
This paper presents a lens-free and portable quantitative phase microscope. This microscope employs a concise off-axis set-up where a dual-pinhole aperture is utilized to generate the reference wave and the object wave. As no lenses or beamsplitters are used in this microscope, the total size of this microscope is only slightly larger than a smart phone, and the cost of this microscope except for the digital camera is about $3000 \mathrm{RMB}$. Even with such small size and low cost, this microscope possesses a lateral resolution of $\sim 1.7 \mu \mathrm{m}$ and an axial accuracy of tens of nanometers.
\end{abstract}

\section{Introduction}

In recent years, digital inline holographic microscopy received great attention for its ability to retrieve three dimensional (3D) information with simple set-up. As a result, diverse outstanding portable holographic systems showed up. One representative work is done by H.J. Kreuzer's group [1-5]. Their systems utilize Gabor in-line set-up to trace 4D trajactories of different biological samples with micrometer resolution. Owing to their sophisticated system and well-designed reconstruction process, the image quality of their system is better than other inline systems [6-8]. However, even though they claimed that their system could also retrieve the phase profile of the sample, they seldom displayed the phase reconstructions. Another outstanding work is developed by A. Ozcan's team [911]. Unlike in traditional Gabor inline holographic microscope, the distance between the sensor and the sample is much smaller than the distance between the pinhole and the sample in their systems. An array of LEDs or a displacement stage is used to capture multiple low-resolution holograms with different illumination conditions. Those holograms are then reconstructed to an amplitude image and a phase image of the sample with high resolution and extended field of view. The dynamic performance of their system, however, is sacrificed for those outstanding features.

In 2014, we presented a concise off-axis holographic technology where off-axis holograms could be captured with inline-like set-up [12]. By adopting that technology, in this paper we demonstrate a low-cost portable holographic microscope for imaging transparent samples. This portable microscope has dimensions of $\sim 13 * 11 * 7 \mathrm{~cm}$ which can be easily held by hands. The performance of

\footnotetext{
a e-mail: yhliu@mae.cuhk.edu.hk
}

this microscope were demonstrated experimentally using an amplitude resolution target and a pure phase object respectively. The results show that this lens-free microscope has a lateral resolution of $\sim 1.7 \mu \mathrm{m}$ and an axial accuracy of tens of nanometers. Besides, a reconstruction program utilizing Graphics Processing Unit (GPU) was written so that both amplitude profile and phase profile of the sample could be reconstructed with a midrange multimedia laptop in real time.

\section{System design}

Fig. 1 demonstrates the schematic diagram and a photograph of our portable microscope. From the schematic diagram, it can be seen that this microscope is mainly composed of a low-cost laser diode (SANWU, LD-T650H00, wavelength: $650 \mathrm{~nm}$, measured power: $\sim 120 \mathrm{~mW}$ ), a lithium battery, a dual-pinhole aperture (National aperture, diameter: $3 \mu \mathrm{m}$, pinhole-pinhole distance: $1 \mathrm{~mm}$ ), a digital camera (XIMEA, MQ042MG-CM, resolution: 2048*2048, Pixel size: $5.5 \mu \mathrm{m}$ ) and a 2D manual linear stage (Newport, consists of two M-MT-X and one M-MT$\mathrm{AB} 2$, travel: $1 \mathrm{~cm}$ ). The shell of this microscope was printed using 3D printing technique and was then sprayed to black in order to prevent laser beam leakage and lower the experimental noise received by the digital camera.

During the operation of this portable microscope, the laser diode keeps projecting a light spot on the dualpinhole aperture. This light spot has a diameter of several millimeters and covers the two pinholes on the aperture. The aperture acts as a spatial filter which filters the light source into an object wave and a reference wave. The object wave illuminates the sample while the reference wave is designed to illuminate a clean area of the sample. The two waves then illuminate the images sensor and create

This is an Open Access article distributed under the terms of the Creative Commons Attribution License 4.0, which permits unrestricted use, distribution, and reproduction in any medium, provided the original work is properly cited. 


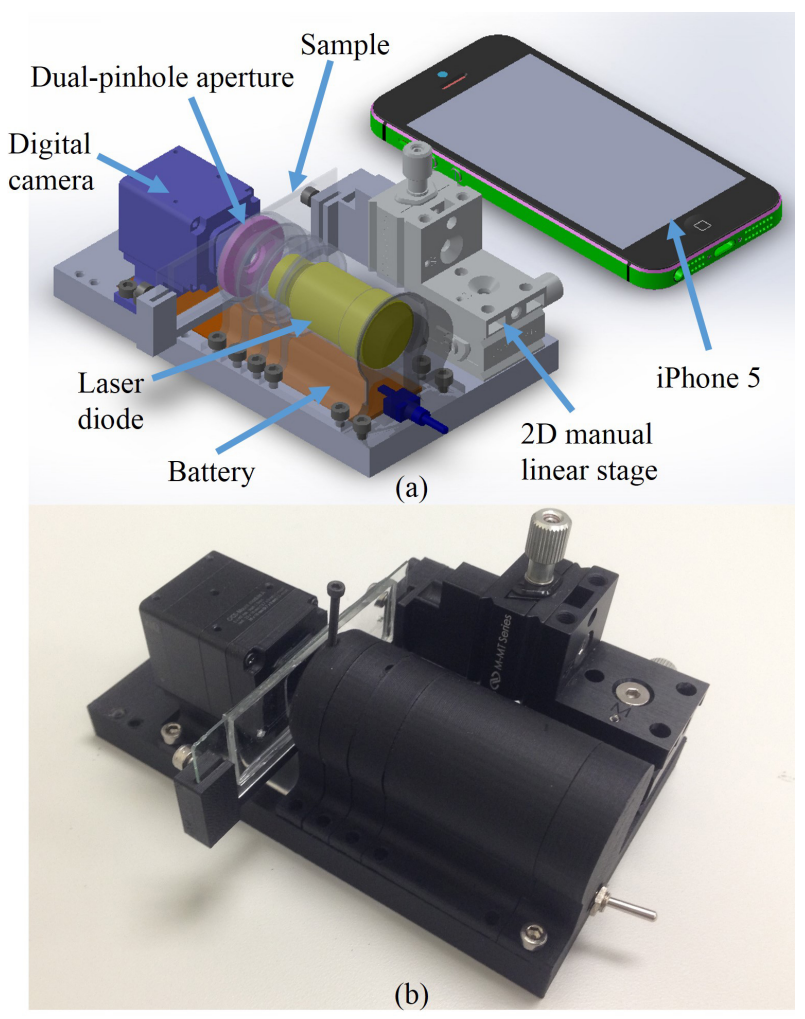

Figure 1. (a) The diagram and (b) the photo of our portable microscope. A model of iPhone5 is provided to give intuitive perception of the size of the portable microscope.

an off-axis hologram. Subsequently, this hologram is captured by the digital camera and sent through a USB 3.0 connection to a PC for reconstruction .

In our system, the diameter of the pinholes should be carefully designed as it influences two major aspects of the image quality. On one hand, the size of the pinholes determines the illumination numerical aperture (NA) of the system, which is one of limiting factors of the lateral resolution of the system. If the diameter of the pinholes is too large, reconstructions will be retrieved with poor lateral resolution no matter how other parameters of the system are changed. On the other hand, the size of the pinholes affects the intensity of the hologram. If the size of the pinholes is too small, the intensity of the hologram will decrease dramatically, which will lead to striking speckle noise in the reconstructions. In order to balance the tradeoffs between the intensity of the hologram and the resolution of the system, $3 \mu \mathrm{m}$ is chosen as the diameter of the pinholes based on our experimental results. In this way, bright holograms could be captured with an exposure time of $10 \mathrm{~ms}$ and the pinholes could provide an illumination NA of $\sim 0.21$.

The distance between the sample and the digital sensor is designed as $26 \mathrm{~mm}$ so that the detection NA matches the illumination NA. As a consequence, theoretically the system could obtain a lateral resolution of $\sim 1.5 \mu \mathrm{m}$. The sample to pinhole distance is designed as $\sim 2 \mathrm{~mm}$, which gives a magnification factor of $\sim 14$.

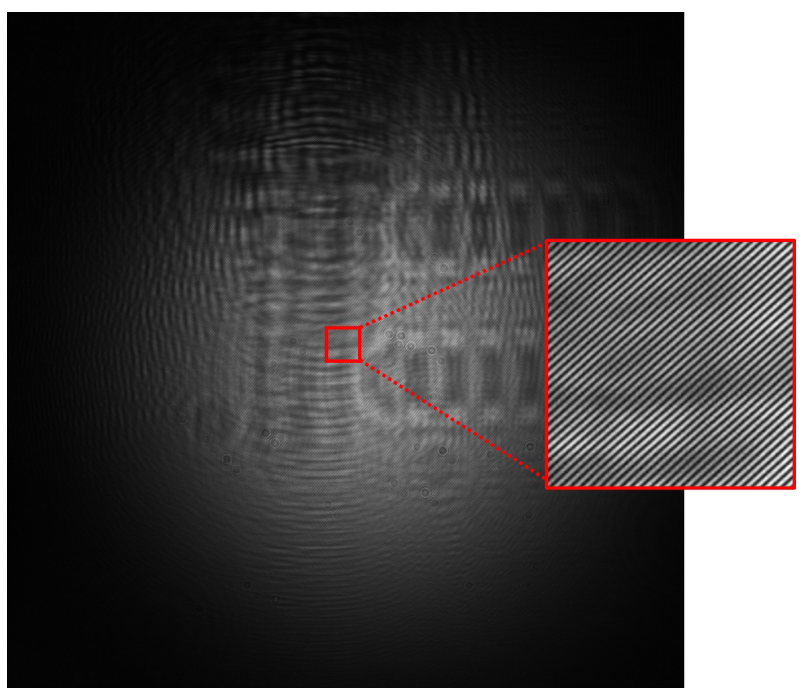

(a)

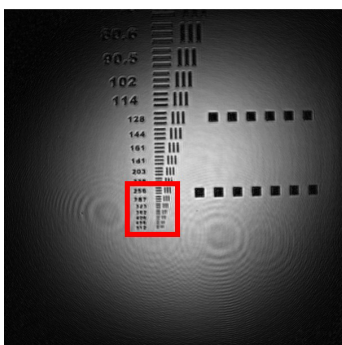

(b)

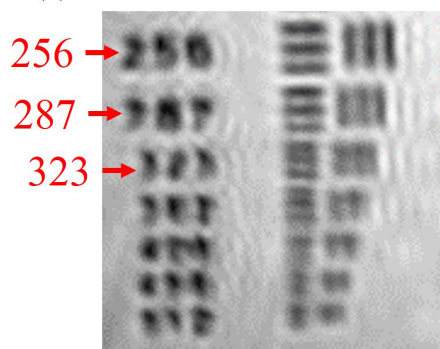

(c)
Figure 2. Lateral resolution test of the system. (a) Captured hologram. (b) Reconstructed amplitude image. (c) Enlarged image of the highlighted part in (b). The red numbers indicate the line density of the corresponding elements of USAF 1951. The unit here is line $/ \mathrm{mm}$.

\section{Experimental results and discussions}

In order to test the lateral resolution of our system, an amplitude resolution target (USAF $1951 \mathrm{~T}-22$ ) was imaged using this portable microscope. Fig. 2 (a) illustrates the resultant hologram where high frequency fringes show up. Fig. 2 (b) and (c) display the amplitude reconstruction of this hologram. Elements with a line width of $1.7 \mu \mathrm{m}$ are resolved which implies that the resolution of our system is about $1.7 \mu m$.

Another hologram captured using our portable microscope is displayed in Fig. 3 (a). In this hologram, we imaged a pure phase object that is made up of polydimethylsiloxane (PDMS). The height difference of this phase object is calibrated as 120 nm using the Alpha-Step surface profiler. Fig. 3 (b) shows the reconstructed phase image of this hologram. The surface profile of the sample could be easily calculated through multiplying a scaler by the unwrapped phase profile. Fig. 3 (c) demonstrates the resultant surface profile of the highlighted line in Fig. 3(b). Here, the measured height difference of the phase object using our system is $118.8 \mathrm{~nm}$, and the standard deviations of the three parts of the line are all below $15 \mathrm{~nm}$.

What's more, a GPU reconstruction program was written to ensure that this portable microscope could recon- 


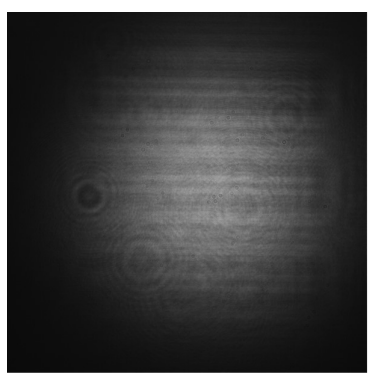

(a)

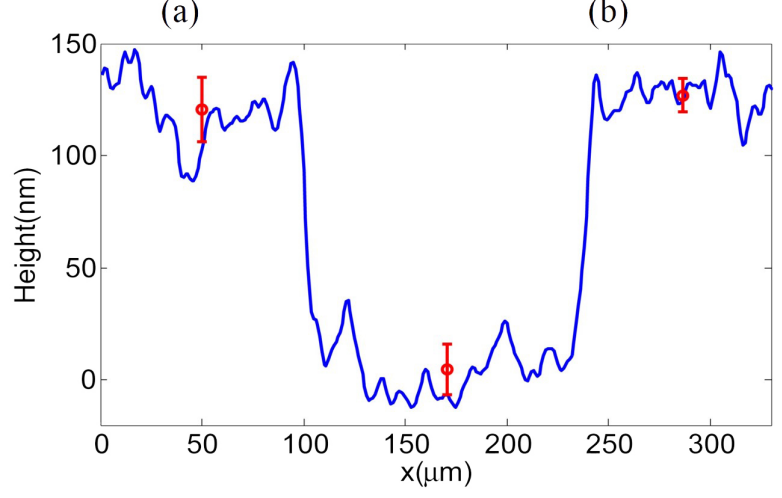

(c)

Figure 3. Axial resolution test of the system. (a) Captured hologram. (b) Reconstructed amplitude image. (c) Surface profile of the highlighted line in (b). The red error bars illustrate the means and the standard deviations of three part of this measurement. 1.4 is adopted as the refractive index of the PDMS.

struct the 3D image of the sample outside the laboratory in real time. The experiment was carried out using a midrange multimedia laptop (Fujitsu, LH772, CPU: Intel(R) Core(TM) i7-3612QM, GPU: NVIDIA GeForce GT $640 \mathrm{M}$ LE). The result shows that our system could reconstruct and display the 3D image of the sample in real time with a frame rate of over $8 \mathrm{~Hz}$. We also tested the reconstruction program with a top-level GPU such as NVIDIA GeForce GTX 690. And the reconstruction frame rate can reach over $100 \mathrm{~Hz}$.

As we only use one wavelength in this system, the axial measurement range of the system are limited within several microns if the measured phase gradient of the sample is larger than $\pi$. In the future, we will develop multiwavelength systems to increase the measurement range and accuracy of the system and retrieve $3 \mathrm{D}$ color images of the sample.

\section{Conclusion}

In this paper, we have demonstrated a low-cost portable holographic microscope which can retrieve the quantitative phase profile as well as the amplitude profile of a transparent sample. The resolution and the dynamic performance of this lens-free microscope were tested. Owing to its cost-effectiveness and portable design, this microscope could be widely used in biological and MEMS applications.

\section{Acknowledgement}

This work is supported in part by the Hong Kong Innovation and Technology Fund via project ITS/089/13, State Key Laboratory of Robotics and System (HIT) via Self-Planned project NO. SKLRS201401B and the Shenzhen Research and Development Fund via project JCYJ20140417172417120.

\section{References}

[1] W. Xu, M. Jericho, I. Meinertzhagen, H. Kreuzer, Proceedings of the National Academy of Sciences 98, 11301 (2001)

[2] W. Xu, M. Jericho, I. Meinertzhagen, H. Kreuzer, Applied optics 41, 5367 (2002)

[3] W. Xu, M. Jericho, H. Kreuzer, I. Meinertzhagen, Optics letters 28, 164 (2003)

[4] J. Garcia-Sucerquia, W. Xu, S.K. Jericho, P. Klages, M.H. Jericho, H.J. Kreuzer, Applied Optics 45, 836 (2006)

[5] J. Garcia-Sucerquia, W. Xu, M. Jericho, H.J. Kreuzer, Optics Letters 31, 1211 (2006)

[6] V. Kebbel, H.J. Hartmann, W.P. Jüptner, Application of digital holographic microscopy for inspection of micro-optical components, in Lasers in Metrology and Art Conservation (International Society for Optics and Photonics, 2001), pp. 189-198

[7] S. Pu, D. Allano, B. Patte-Rouland, M. Malek, D. Lebrun, K. Cen, Experiments in Fluids 39, 1 (2005)

[8] T. Shimobaba, Y. Taniguchi, A. Shiraki, N. Masuda, T. Ito, Portable and low-cost digital holographic microscopy using web camera, point light source LED and open-source libraries, in Biomedical Optics (Optical Society of America, 2012)

[9] A. Greenbaum, N. Akbari, A. Feizi, W. Luo, A. Ozcan, PloS one 8, e76475 (2013)

[10] A. Greenbaum, Y. Zhang, A. Feizi, P.L. Chung, W. Luo, S.R. Kandukuri, A. Ozcan, Science translational medicine 6, 267ra175 (2014)

[11] Y. Zhang, A. Greenbaum, W. Luo, A. Ozcan, Virchows Archiv pp. 1-5 (2015)

[12] Y. Lu, Y. Liu, T.K. Lau, Optics letters 39, 4549 (2014)

[13] M. Lee, O. Yaglidere, A. Ozcan, Biomedical Optics Express 2, 2721 (2011) 